\title{
5. THE DISTRIBUTION OF DARK NEBULAE IN \\ LATE-TYPE SPIRALS
}

\author{
B. T. LYNDS
}

Steward Observatory, University of Arizona, Tucson, Ariz., U.S.A., and

Institute of Theoretical Astronomy, Cambridge, England

\begin{abstract}
Seventeen Sc- galaxies have been studied in order to determine the distribution of dark nebulae within them. $\mathrm{H} \alpha$ plates were used to compare the distribution of obscuring material with the location of Hil regions, and it was found that the Hil regions are always tangent to or imbedded in regions of high obscuration. Sandage's conclusions are confirmed that strong, regular dust lanes exist in the central regions of these galaxies and wind outward on the inside edge of the two prominent arms. Characteristic dimensions of the dust lanes are noted, and secondary dust characteristics are described.
\end{abstract}

The most detailed notes on the distribution of interstellar dust in galaxies have been given by Sandage and are to be found in the description of the Hubble classification and in the captions for the illustrations of the Hubble Atlas (Sandage, 1961). In this publication, Sandage points out that the spiral pattern for Sc-galaxies first becomes apparent near the center of such systems as two principal dust lanes; luminous spiral arms appear only at larger distances from the nucleus, at which distances the two principal dust lanes wind out along the inside of the two most luminous outer arms. After winding out for nearly half a revolution, these two principal arms branch into segments which continue to spiral outward to form the multiple-arm structure. Furthermore, Sandage points out that the dust is not confined to these lanes but rather is seen in spiral patterns over the entire face of such systems.

Several detailed studies of individual galaxies have been made in which the distribution of the interstellar dust is discussed, but the emphasis has usually been on the determination of the sense of direction of the motion of spiral arms. Other than the report of Sandage, no detailed studies of the sizes and locations of dark nebulae in galaxies are known to the author. Because of the recent revival of interest in the role played by the dust, a systematic analysis of the sizes and distributions of the dark material in external galaxies should be of interest.

Through the courtesy of the Mount Wilson and Palomar Observatories, the plate collection of 100- and 200-inch photographs of galaxies constitutes the raw material for this study. All galaxies in the Shapley-Ames Catalogue were visually inspected, and a sample of these objects was selected for detailed measurement. The $x$-, $y$ coordinate measuring machine of the Mount Wilson Observatory was used to scan across each sample galaxy and to record the positions of the dark and bright spiral features. This report is confined to results obtained from a study of seventeen late-type spirals, classified as Sc- by Holmberg (1958).

Although interstellar dust is most easily detected in spiral systems whose plane of symmetry lies nearly in the line of sight, it is very difficult to determine the exact location of the dust in such systems. Therefore, for this study, the galaxies selected 
are those seen nearly face-on for which there is some degree of certainty of identification of the dust clouds. The results given here therefore represent data for those Sc-galaxies in which the dust is readily apparent and relatively easily measured. It is a happy coincidence that such Sc galaxies appear to be the most commonly observed and may reflect average properties of Sc-systems in general in spite of the fact that the sample of 17 studied was in no sense unbiased.

The usual criteria for identification of a dark nebula in any stellar system are firstly, the absence of stars relative to the surrounding area, and secondly, the ability to detect a relatively well-defined boundary of the nebula. In the case of an external spiral galaxy, one can often use these two criteria with as great a certainty as one can in our own Milky Way System. The identification becomes more difficult in the inter-arm regions of spirals and in the outer zones of a galaxy beyond its luminous arms. An effort was made to confine the data to dark regions whose presence would be difficult to interpret other than to say that they are true obscuring clouds.

It was initially hoped that the brightest HII regions of a galaxy would define the spiral pattern against which the positions of the dark nebulae could be compared. Therefore, in this preliminary report, those galaxies (which met the first requirement of containing easily-detected dark clouds) which have available $\mathrm{H} \alpha$ interference-filter photographs in the Mt. Wilson and Palomar plate collection were selected. Perhaps the most interesting result of this study is the confirmation of the oft-quoted statement that there is a 'one-to-one correspondence' between the presence of dust and the presence of young stars. On the basis of the sample studied here, it is concluded that the brightest $\mathrm{H}$ II regions of a galaxy are always found either next to or imbedded in regions of high obscuration. The converse of the statement is not true; many regions of equally dense obscuration contain no $\mathrm{H}$ il regions. It is also the conclusion of this study that the primary dust lanes of a galaxy better define the spiral pattern than do the Hil regions, which often are found on the inside edge of a bright spiral arm but may be found on the outside edge or somewhere within the luminous arm where a 'feather' of a dark lane cuts across the arm. Occasionally, $\mathrm{H}_{\text {II }}$ regions are even found in heavily-obscured inter-arm areas (if the arms are defined by the regions of relatively uniform luminosity).

Figures 1-5 contain the distribution of the dark nebulae within those galaxies for which $\mathrm{H} \alpha$ photographs were available. The lightly-shaded areas represent the dust lanes of the galaxy; the heavy black marks are the locations of the brightest $\mathrm{H}$ il regions.

When one studies the sketches of Figures 1-5; or, better, when one examines the photographs themselves (see, for example the prints of the Hubble Atlas), certain regular patterns of the distribution of dust become apparent. The two most striking features are those pointed out by Sandage: the existence of very strong dust lanes in the central regions and the presence of dark lanes along the inside edge of the two luminous arms. These latter primary dust lanes can occasionally be traced through more than $360^{\circ}$, that is, through a 'first' and 'second' winding of the arms. In addition to these characteristics, thin dust lanes are also found cutting their way across a bright arm with pitch angles of about $50^{\circ}$ relative to the primary lanes. 
Once such 'feathers' have been traced across a luminous arm, they are no longer visible against a bright background. There is, however, some evidence that an extensive area beyond a luminous arm also contains dust condensations. Another, smaller scale, characteristic of dark nebulae in these systems is the appearance of nearly

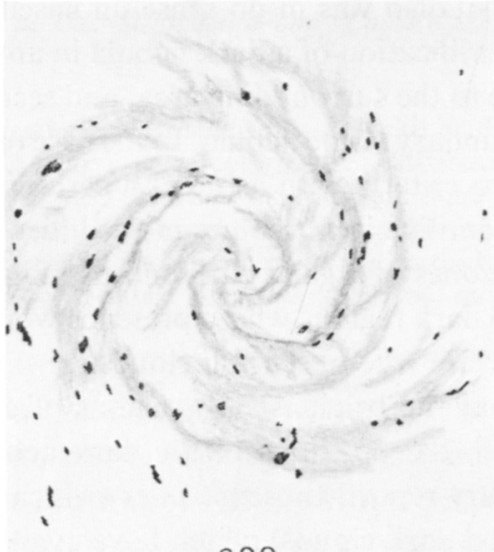

628

Fig. 1. NGC 628. This is the most regular of the Sc-galaxies studied. The two primary dust arms can be relatively easily traced through a first and second winding. The luminous arms are found sandwiched between the dark nebulae of the sketch.

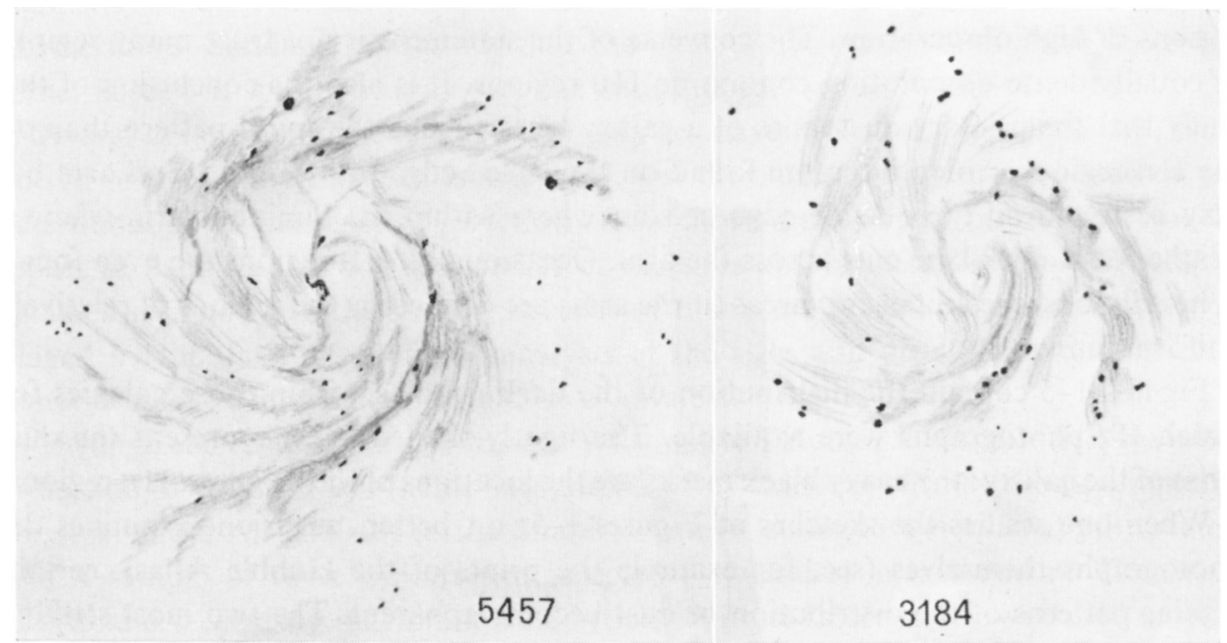

Fig. 2. NGC 5457. M 101 is the prototype of the special subgroup of Sc-galaxies which shows the most regular dust patterns. The luminous arms are thinner than those of NGC 628, which belongs to the same subgroup. Sandage has pointed out that in M 101 the dust lanes appear as separate segments which combine to form a spiral pattern. NGC 3184: No H $\alpha$ interference filter photograph was available for this galaxy, but the H II regions listed by Hodge (1969) were identified on the 100 -inch 103aO photograph. Hodge's data were based on 48-inch Schmidt photograph, so that the central regions are incomplete in the $\mathrm{HII}$ identifications. The dust in this galaxy is more difficult to detect. 
circular clouds which often contain a very bright central knot. These bright knots are frequently not $\mathrm{H}_{\text {II }}$ regions but may be one or more bright blue stars.

Table I lists the representative sizes of the characteristic interstellar features. The distances $(m-M)$ and radii $(R)$ were taken from Holmberg (1964). The galaxies are

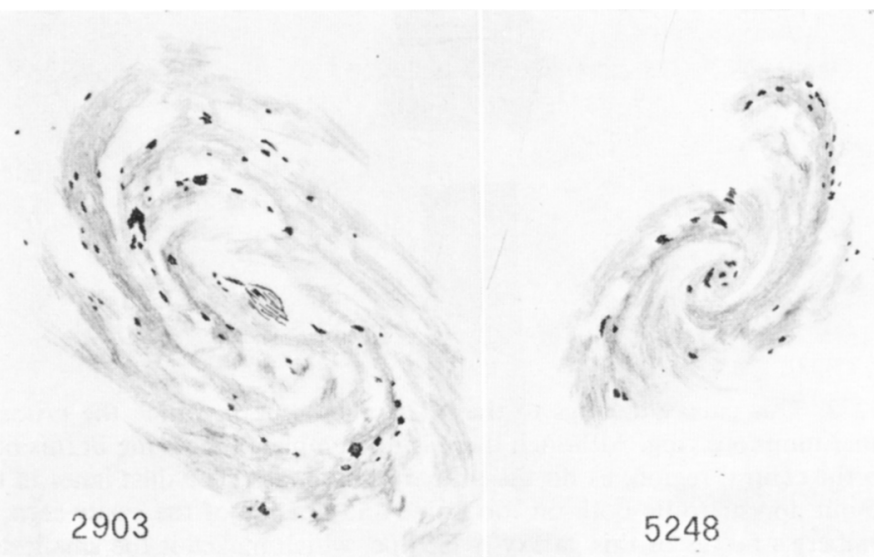

Fig. 3. NGC 2903. These two galaxies are viewed more highly inclined to the line of sight. NGC 2903 belongs to the NGC 253 subgroup of Sc-galaxies which has ill-defined spiral arms and more chaotic dust. For the subgroup, spiraling dark lanes are still quite apparent over the entire galaxy. - NGC 5248. This galaxy is of the NGC 1637 type, whose luminous arms are not as well-defined as those of M 101 subgroup. Nevertheless, this subgroup shows the same characteristic dust pattern as does the M 101 type.

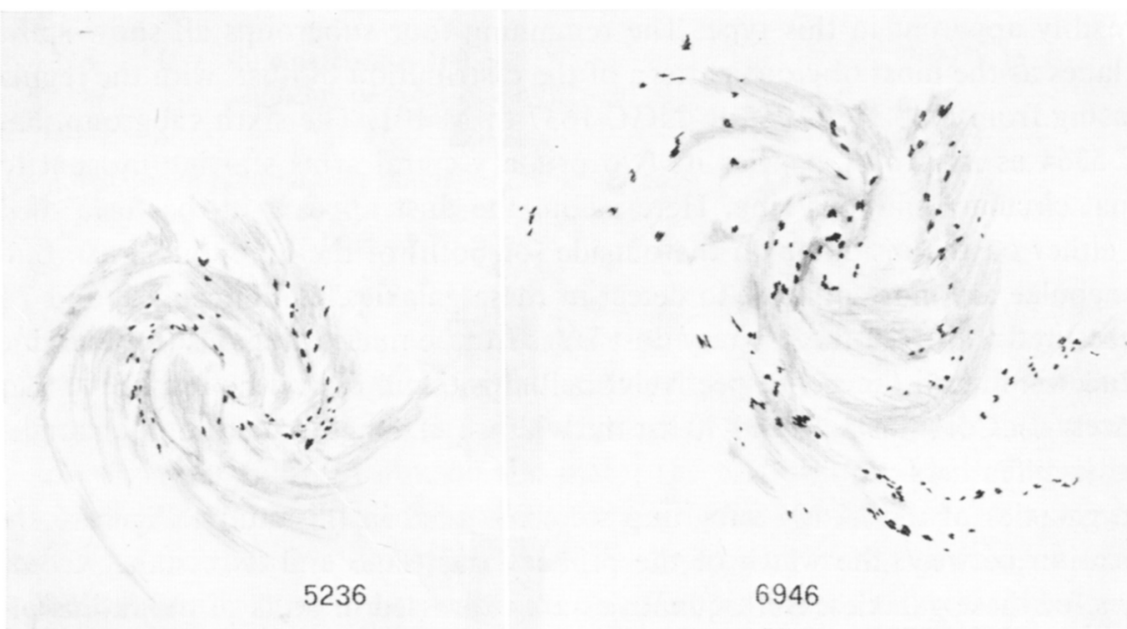

Fig. 4. NGC 5236. The spiraling dust segments in this very open galaxy are its most dominant feature. The arms appear to be exceptionally rich in $\mathrm{HII}$ regions and are quite obviously closely associated with the dust. - NGC 6946. The dust pattern in this galaxy is similar to NGC 5236 but more difficult to measure and the luminous arms are more difficult to trace. The 48-inch Schmidt print of this galaxy strongly suggests that obscuration exists in the outer regions beyond the luminous primary lanes, as sketched in the figure. 


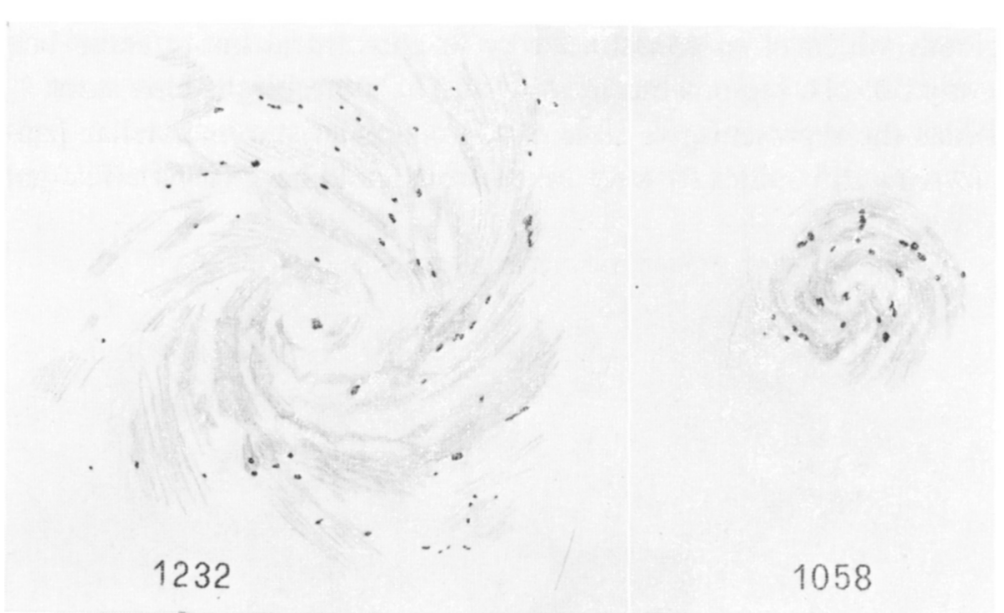

Fig. 5. NGC 1232. This galaxy belongs to the NGC 5364 type in which the primary arms begin tangent to an inner luminous ring. Although there is no complete inner ring in this object, the arms begin tangent to the central region, as do the primary dust lanes. The dust lanes in this galaxy are difficult to trace but appear to lie both on the inside and outside of the easily-seen bright arms. NGC 1058. Holmberg's radius of this galaxy is $6.6 \mathrm{kpc}$, which makes it the smallest galaxy in this study. The primary dust lanes produce the spiral appearance of this galaxy.

divided into the Sc subgroups defined by Sandage (1961). Five of his six major subgroups are represented in the table; only the NGC 4395-type (having very chaotic and ill-defined spiral arms) is not included. NGC 2403, like M 33, has obvious dust patches, but the dominant spiraling dark lanes of the other types of Sc systems are not readily apparent in this type. The remaining four subgroups all show spiraling dark lanes as the most obvious pattern of the distribution of dust, with the regularity increasing from type NGC 253 to NGC 1637 to M 101. The sixth subgroup, having NGC 5364 as its prototype, has its two primary spiral arms starting tangent to an external circular luminous ring. Here again, the dust appears in the form of dark lanes either on the inside or on the outside (or both) of the luminous arms, but the dark nebulae are more difficult to detect in these galaxies. Columns 4, 5, and 7 give the measured widths of the primary dust lanes for the nuclear regions $(n)$, 1st winding and 2nd winding distances, respectively; columns 6 and 8 give the respective nuclear distances $(d)$. Columns 8,9 , and 10 list the widths $(w)$, lengths $(l)$, and pitch angles $(i)$ of the dark feathers.

Ten galaxies of the M 101 subgroup were included in this study. Figure 6 shows the relation between the width of the primary dust lane and its distance from the nucleus for these galaxies. Both quantities are expressed in units of the radius of the galaxy. It appears that there is a general increase in thickness of the primary dust lane as it winds its way out from the nucleus; the overlapping of the points representing first (filled circles) and second (open circles) winding positions indicates that the width depends more on the nuclear distance than on the tightness of the winding of the arms. The lower portion of Figure 6 shows that the measurements of the width of the primary 
TABLE I

Dust measurements in Sc-galaxies

\begin{tabular}{|c|c|c|c|c|c|c|c|c|c|c|c|}
\hline \multirow[t]{2}{*}{ NGC } & \multirow{2}{*}{$m-M$} & \multirow{2}{*}{$\begin{array}{c}R \\
\mathrm{kpc}\end{array}$} & \multicolumn{4}{|c|}{ Primary dust lanes } & \multirow[b]{2}{*}{$\begin{array}{l}d \\
\mathrm{kpc}\end{array}$} & \multicolumn{3}{|c|}{ Feathers } & \multirow[t]{2}{*}{ Plates $^{a}$} \\
\hline & & & $\begin{array}{l}n \\
\mathrm{pc}\end{array}$ & $\begin{array}{l}1 \mathrm{st} \\
\mathrm{pc}\end{array}$ & $\begin{array}{l}d \\
\mathrm{kpc}\end{array}$ & $\begin{array}{l}\text { 2nd } \\
\mathrm{pc}\end{array}$ & & $\begin{array}{l}w \\
\mathrm{pc}\end{array}$ & $\begin{array}{l}l \\
\mathrm{kpc}\end{array}$ & $i$ & \\
\hline \multicolumn{12}{|c|}{ M 101-Type } \\
\hline 157 & 31.7 & 18 & - & 350 & 2.8 & 900 & 7 & 160 & 1.0 & $75^{\circ}$ & $103 \mathrm{aO}$ \\
\hline 628 & 29.7 & 15 & 60 & 100 & 1.1 & 530 & 3 & 60 & 0.5 & 50 & $103 \mathrm{aO}, \mathrm{D}, \mathrm{E} ; \mathrm{H} \alpha$ \\
\hline 2532 & 34.0 & 35 & - & 300 & 2.0 & - & - & - & - & - & $103 \mathrm{aO}$ \\
\hline 3184 & 29.3 & 10 & - & 80 & 0.4 & 190 & 2 & - & - & - & $103 \mathrm{aO}(100$-inch) \\
\hline 4254 & 30.5 & 13 & - & 250 & 1.2 & 320 & 2 & 80 & 0.8 & 45 & $103 \mathrm{aE}$ \\
\hline 4303 & 30.5 & 20 & 130 & 100 & 2.4 & - & - & - & - & - & $103 \mathrm{aO}$ \\
\hline 4321 & 30.5 & 18 & 120 & 270 & 2.4 & 270 & 5 & 130 & 0.9 & 45 & 103aO, D, E \\
\hline 5236 & 28.2 & 8 & 70 & 200 & 1.4 & 300 & 3 & 40 & 0.5 & - & E40 (100-inch), $\mathrm{H} \alpha$ \\
\hline 5457 & 27.7 & 14 & 30 & 50 & 0.6 & 200 & 2 & 40 & 0.2 & 60 & $103 \mathrm{aD}, \mathrm{E} ; \mathrm{H} \alpha$ \\
\hline 6946 & $28.4^{\mathrm{b}}$ & 9 & 40 & 150 & 1.2 & 540 & 3 & 50 & 0.4 & - & $\begin{array}{l}103 \mathrm{aO}(100 \text {-inch }) \\
103 \mathrm{aE} ; \mathrm{H} \alpha\end{array}$ \\
\hline \multicolumn{12}{|c|}{ NGC 1637-Type } \\
\hline 1637 & 29.5 & 9 & & 100 & 0.8 & 130 & 1.3 & & & & $103 \mathrm{aO}$ \\
\hline 5248 & 30.9 & 18 & & & & 210 & 4 & & & 55 & $103 \mathrm{aO}, \mathrm{H} \alpha$ \\
\hline 1058 & 29.4 & 7 & & 80 & 0.2 & 240 & 0.5 & & & & $103 \mathrm{aO}, \mathrm{D}, \mathrm{E} ; \mathrm{H} \alpha$ \\
\hline \multicolumn{12}{|c|}{ NGC 5364-Type } \\
\hline 864 & 31.5 & 13 & & 560 & 2. & 1000 & 4 & & & & $103 \mathrm{aO}$ \\
\hline 1232 & 30.7 & 19 & & & & 640 & 2 & & & 40 & $103 \mathrm{aO}, \mathrm{H} \alpha$ \\
\hline 5364 & 30.1 & 12 & & 330 & 1.9 & 550 & 4 & & & & $103 \mathrm{aO}$ \\
\hline \multicolumn{12}{|c|}{ NGC 253-Type } \\
\hline 2903 & 29.5 & 16 & & $\sim 100$ & 3 & & & & & & $103 \mathrm{aO}, \mathrm{H} \alpha$ \\
\hline \multicolumn{12}{|c|}{ М 33-Туре } \\
\hline 2403 & 27.6 & 14 & \multicolumn{8}{|c|}{$\begin{array}{l}\text { dust patches } \sim 100 \mathrm{pc} \\
\text { segments of lanes of width } \sim 30 \mathrm{pc}\end{array}$} & $103 \mathrm{aO}, \mathrm{D}, \mathrm{E}$ \\
\hline
\end{tabular}

dust lane are not distance-dependent for this sample. Most of the plates measured were 200-inch direct photographs, with a scale of $11.06^{\prime \prime}$ per $\mathrm{mm}$. It is possible to be fairly certain in the identification and measurement of a dark lane which has a thickness equal to or greater than $0.1 \mathrm{~mm}$ on the plate; the observational lower limit to the detection of a dark lane thus corresponds to about one second of arc. The measured thicknesses of the thin feathers of dark nebulae branching from the primary lanes do show a distance effect, as illustrated in Figure 7. The width of the feathers appears to be about one-half that of the primary lane, if only the upper part of Figure 7 is considered. However, the lower part of the figure suggests that although the measured feather widths appear to be greater than the observational lower limit for galaxies whose distance moduli are less than 30 , beyond this distance of $10 \mathrm{Mpc}$ the measurements represent only the selected wider lanes. The dashed line in the lower part of 

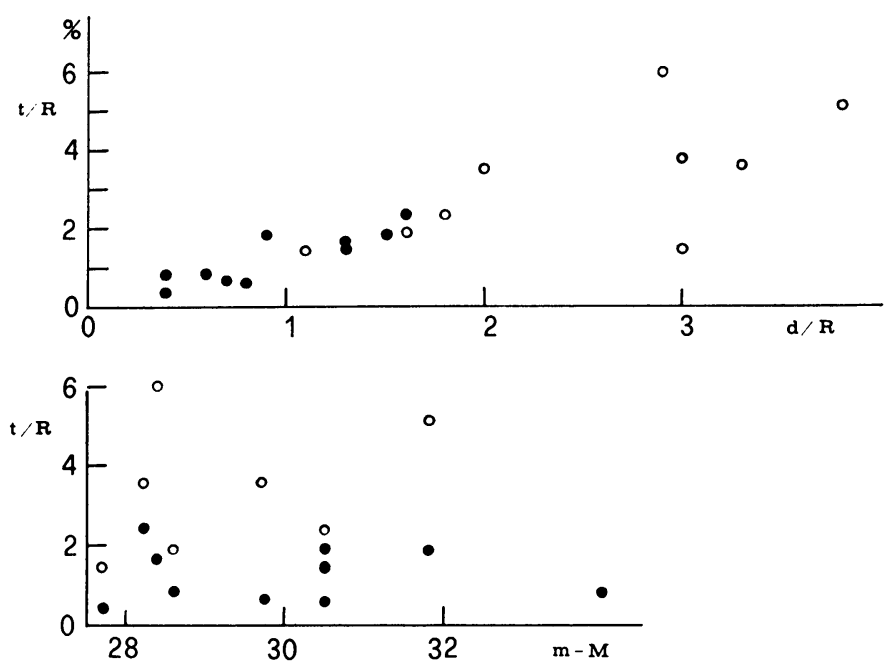

Fig. 6. Variation in width of primary dust lanes with distance from the nucleus (upper) and with distance modulus (lower). The units are in terms of the radius of the galaxy. The ordinate is the percentage width of the primary lane; the upper abscissa is the fractional distance from the center of the galaxy. The lower array of points shows that the measurements are independent of distance for the ten M 101 galaxies studied. The filled circles are measurements of the first winding thicknesses; the open circles are the second winding measurements.

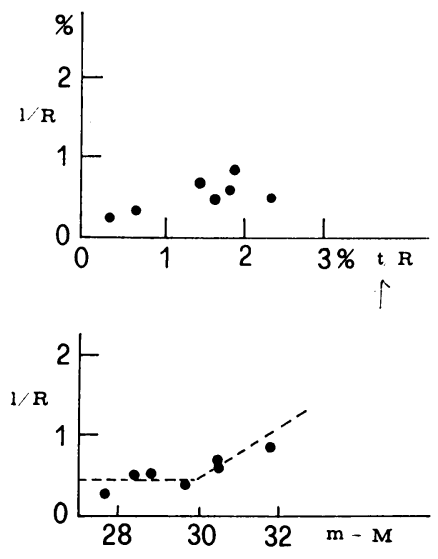

Fig. 7. Variation in width of dust feathers with width of primary lanes (upper) and with distance (lower). The Units are percentage of radius of the galaxy. The dashed curve of the lower diagram represents the expected variation in the measured feather widths if a galaxy of $11 \mathrm{kpc}$ had dark feathers of widths $50 \mathrm{pc}$ and greater.

Figure 7 illustrates this resolution effect and defines the locus of observational points to be expected if a galaxy of radius $11 \mathrm{kpc}$ had feathers of widths $50 \mathrm{pc}$ and greater.

Table I also lists the emulsion sensitivities of the plates measured in this study. For some spirals, scans across the galaxy were made on $\mathrm{O}, \mathrm{D}$, and $\mathrm{E}$ plates. The 
accuracy of positional registration of these plates for intercomparison is about 30 microns, and to this accuracy no difference was found in the sizes of the dark nebulae over the wavelength regions represented by the red and the blue plates. This must mean that the nebulae selected to be measured are of very large optical thickness. Furthermore, in many regions of these galaxies, dark lanes can be seen occulting a luminous spiral arm which itself is several magnitudes above the plate limit. It is therefore concluded that the extinction through the dark clouds whose characteristic sizes are listed in Table I must be greater than about 3 magnitudes.

If 3 magnitudes is adopted as the lower limit to the optical extinction through the dark nebulae and if Holmberg's distances are assumed, then the actual physical sizes of the nebulae may be estimated. If we adopt $15 \mathrm{kpc}$ as an average radius of a M 101-type Sc-galaxy, then Figure 7 may be used to estimate the width of the primary dust lanes. Near the nucleus the lane has a thickness of about $50 \mathrm{pc}$; the width of the lane gradually increases, reaching a value of about $240 \mathrm{pc}$ at a distance of $2 \mathrm{kpc}$ from the center. Unfortunately, we have no means of estimating the $z$ thickness of the cloud, which is the pathlength needed to correspond to the 3 magnitude extinction estimate. If we use the simple model in which the lane is as 'thick' as it is wide, the pathlength may be set equal to the measured width of the lane. In this case we can estimate the lower limit to the grain density within the primary lane. According to Solomon (1969), the grain density of a cloud of 3 magnitudes absorption is given by

$$
\varrho_{\mathrm{g}}=10^{-4} / \mathrm{L} \mathrm{g} \mathrm{cm}^{-3},
$$

where $L$ is the pathlength in $\mathrm{cm}$ through the cloud. For our model lane the grain densities must be greater than $7 \times 10^{-25} \mathrm{~g} \mathrm{~cm}^{-3}$ for the nuclear regions and $4 \times 10^{-25} \mathrm{~g} \mathrm{~cm}^{-3}$ at a distance of $2 \mathrm{kpc}$ from the center. This assumes that the extinction is produced by the same type of particle which exists in our galaxy. If we further assume the usual 100: 1 ratio of gas to dust, we find an interstellar density greater than about $10^{-23} \mathrm{~g} \mathrm{~cm}^{-3}$ throughout the winding of the primary dust lane. In an analogous manner, the mass of a typical feather of obscuration must be greater than $10^{6}$ solar masses. Such a lane of dark nebulosity crossing a luminous arm meets the requirements of Spitzer and Schwarzschild (1953) who predicted similar interstellar clouds on dynamical arguments related to stellar velocity dispersions.

In summary, the seventeen Sc-galaxies studied here indicate that the spiral pattern of these galaxies is revealed by the distribution of the interstellar dust. The dominant lanes of obscuration evident in most of these systems probably have densities comparable to those of the luminous arms themselves. Although each galaxy on close inspection has many individual features peculiar to itself, the overall pattern of dust as defined in this study seems to be characteristic of such systems.

\section{Acknowledgements}

The author is greatly indebted to Dr. Allan Sandage for his generous assistance in making the Palomar plate collection available for this study, and to Dr. Horace Babcock 
for permission to use the Mt. Wilson and Palomar Observatories equipment. The manuscript was prepared while the author was a visitor at the Institute of Theoretical Astronomy, and it is a pleasure to acknowledge the hospitality of Prof. F. Hoyle and his associates.

\section{References}

Hodge, P. W.: 1969, Astrophys. J. Suppl. Ser. 18, 73.

Holmberg, E.: 1958, Medd. Lunds Astron. Obs., Ser. II, No. 136.

Holmberg, E.: 1964, Ark. Astron. 3, 387.

Sandage, A.: 1961, The Hubble Atlas of Galaxies, Carnegie Institution of Washington, Washington. Solomon, P.: 1969, private communication.

Spitzer, L. and Schwarzschild, M.: 1953, Astrophys. J. 118, 106. 